\title{
La televisione nella prosa di Aldo Nove
}

\section{Gabriella Macrì}

\section{OpenEdition \\ Journals}

Edizione digitale

URL: http://journals.openedition.org/cei/105

DOI: $10.4000 /$ cei. 105

ISSN: 2260-779X

\section{Editore}

UGA Éditions/Université Grenoble Alpes

\section{Edizione cartacea}

Data di pubblicazione: 15 juin 2010

Paginazione: 63-72

ISBN: 978-2-84310-168-7

ISSN: 1770-9571

Notizia bibliografica digitale

Gabriella Macrì, «La televisione nella prosa di Aldo Nove», Cahiers d'études italiennes [Online], 11 | 2010, online dal 15 décembre 2011, consultato il 26 mars 2021. URL: http://journals.openedition.org/cei/105 ; DOI: https://doi.org/10.4000/cei.105 


\title{
LA TELEVISIONE NELLA PROSA DI ALDO NOVE
}

\author{
Gabriella Macri \\ Université de Salonique
}

Nel 1996 gli scrittori «cannibali» o pulp venivano presentati nell'antologia einaudiana Gioventù cannibale che fu subito un caso editoriale ${ }^{I}$. I riferimenti, i luoghi e i temi narrativi, i motivi d'ispirazione della scrittura "cannibale» (espressi con un registro linguistico tendente al basso, alla parola-merce) riflettono il mondo contemporaneo, la subcultura popolare, il trash, la pubblicità televisiva, la cultura massmediatica e cinematografica del kitsch.

[La televisone] proprio attraverso quei testi, assume un ruolo inedito nella storia della letteratura italiana: per la prima volta invade i territori della narratività in modo pervasivo e nell'accezione multipla di canale comunicazionale, codice linguistico, luogo di formazione dell'immaginario, elemento creatore di simboli e miti. In molti autori il paradigma narrativo appare rivoluzionato da un continuo dialogare con la civiltà televisiva, con ostilità e impugnando le armi del paradosso, dell'ironia e del rovesciamento grottesco. (Mondello, 2007, p. 77.)

In un capitolo del suo libro-inchiesta sul precariato Mi chiamo Roberta, ho 42 anni, guadagno 250 euro al mese..., Nove giustifica il motivo di esistere della letteratura pulp italiana e traccia una linea di collegamento tra essa e la realtà filtrata attraverso le trasmissioni televisive. Scrive in proposito:

Il fenomeno letterario pulp nacque, a metà degli anni Novanta, come testimonianza letteraria $[\ldots]$ di un mondo ormai ridotto a slogan, pubblicità, tette ma anche violenza, gratuita e spettacolare. Con un linguaggio sempre più aggressivo e vacuo.

(Nove, 2006, p. 42.)

I. Mario Barenghi (1999, pp. 288-289) fa una distinzione tra letteratura pulp e «cannibale» sottolineando quanto quest'ultima definizione possa connotare meglio del "fuorviante $p u l p$ » le prerogative della "poetica» dei giovani scrittori esordienti nell'antologia einaudiana. 
Per Nove il pulp è dunque la risposta letteraria alla condizione socioculturale di un mondo all'apparenza confortevole ma profondamente aggressivo. Nell'ultimo decennio, però, la televisione ha ridotto sempre più la capacità di trasmettere valori etici o culturali, con una inesorabile riduzione dell'osmosi tra pulp e mass media. Oggi, conclude lo scrittore, questo genere letterario sembra non avere più motivo di esistere:

La televisione sta rientrando nel suo ruolo di elettrodomestico. Quello di Orwell è un ciclo concluso. [...] Lo stesso credo si possa dire, in scala ovviamente minore, per il pulp, come suo riflesso letterario critico. (Nove, 2006, p. 42.)

Nei 48 racconti di Woobinda (1996, ampliato poi in Superwoobinda nell'edizione del 1998) Nove vuole indurre il lettore a riflettere sulla futilità di certe trasmissioni televisive odierne. Si pensi al racconto «I programmi dell'accesso", dove accusa la televisione di alienazione dell'individuo, di manipolazione della vita dello spettatore anche con i programmi educativi; o a "Protagonisti» in cui gli attanti scandiscono la giornata in base all'orario d'inizio dell'omonima trasmisione televisiva, che diventa un momento in cui s'illudono di comunicare con qualcuno.

I racconti sono connotati da un linguaggio stereotipato, una parodia di quello televisivo ("Protagonisti è il programma che ti mette al centro della scena», Nove, 1998, p. I27). Molte espressioni sono tipiche delle formule televisive («Vidi un ragazzo. E una ragazza. Una moto. La campagna. Nei loro sguardi la gioia di essere giovani. Neocibalgina», ibid., p. II7), della lingua orale («Una giornata di lavoro ti entra nelle vene, sai. Non capisci più quello che fai, sei lì e guardi la tele», ibid., p. 80), talvolta si incontrano regionalismi lombardi ( Gli Smarties sono una delle cose più belle di quando ero un attimino piccolo», ibid., p. 97; «Dissi a Marcello di non fare il pirla», ibid., p. 50). Gli attanti hanno una personalità distorta o poco sviluppata, sono «interamente plasmati dagli idoli e feticci della pubblicità, dagli orrori, dai crimini efferati di cui i notiziari televisivi parlano qutodianamente» (Barilli, 2000, p. II5): manifestano il loro disagio con la violenza, con la cultura del consumismo e con fantasie erotiche, spesso scatenate dalla visione di un film porno. Il mezzo televisivo condiziona il loro quotidiano giocando un ruolo fondamentale sulla loro fragilità psichica con i contenuti massivi dei palinsesti. Non sembrano avere passato né futuro ma vivono soltanto nel presente, possiedono una scarsa memoria storica e personale, presentano poche caratteristiche caratteriali, vivono l'attimo, anzi lo bruciano per passare a quello successivo. Gli episodi che li vedono protagonisti non sono collocabili o individuabili in un cronotopo ma restano sospesi in una loro realtà che nulla ha a che fare con 
la realtà storica; semmai con quella plasmata dalla televisione: insomma, sembrano essere usciti dai format televisivi e pulsare di vita propria. Anche la donna diventa proiezione dello stereotipo femminile dei programmi televisivi, e spesso supera il pudore e il riserbo propri della vita domestica per essere protagonista (o vittima) di insolite pratiche sessuali. In ogni racconto la televisione è messa in relazione a un determinato argomento: giovani, politica, cultura, letteratura, pornografia, infanzia, informazione, sesso, diventa ostentazione di ricchezza. Gli episodi si interrompono bruscamente con una parola incompleta, così come lo zapping interrompe la visione di una trasmissione. Nel 1996 su Pulp Nove giustificava la sua scelta stilistica con la seguente (ormai nota) dichiarazione:

Il mio scopo dichiarato era quello di riportare il ritmo dello zapping in letteratura, scrivere televisivamente ciò che è breve, veloce e spezzato. Ė stato un misto di scelta letteraria e... come dire... di gratificante comodità, perché così si vive e così si parla. Non dovevo fare il finale e questo anziché diventare uno svantaggio ha finito per conferire coerenza interna al discorso. La scelta del microracconto è stata quasi obbligata: è la forma più congeniale al genere, quella letteratura televisiva di cui si diceva prima. (Cito da Gervasutti, 1998, pp. 4I-42.)

Tra Woobinda e gli ultimi due romanzi si interpone la scrittura di Puerto Plata Market (1997), che segna un ritorno/recupero dei canoni narrativi della tradizione italiana. Ormai Nove abbandona le vesti dello scrittore "cannibale» (in varie situazioni aveva dichiarato che questa definizione non aveva più motivo di esistere) che a lui risulta inadeguata. Nel romanzo, dove ciascun capitolo costituisce un episodio a sé, gli attanti si muovono in modo più autonomo, sebbene ogni loro comportamento sia, ancora una volta, condizionato dal consumismo, anche nell'amore.

Rispetto a Woobinda la televisione acquista, in Puerto Plata Market, una duplice valenza. Nei primi capitoli è funzionale all'io narrante Michele ${ }^{2}$ che, in crisi dopo la fine del rapporto con Marina, cerca di ritrovare sé stesso ripercorrendo alcuni momenti dell'infanzia e dell'adolescenza. Ricordando alcune trasmissioni viste da bambino, Michele proietta memoria e sensazioni personali in una dimensione collettiva: rievoca il ciclo di Sandokan, lo Zecchino d'oro, gli episodi di Speedy Gonzales, i documentari sui bambini del Biafra, le allegre avventure di Pippi Calzelunghe. La notizia del ritrovamento di Aldo Moro s’intreccia coi personaggi del suo mondo infantile:

2. Il nome e l'origine varesina richiamano l'omonimo protagonista di Ok il prezzo è giusto, pubblicato su Gioventù cannibale.

3. "Aldo Moro e Pippi Calzelunghe erano per me due cose completamente diverse. [...] Mi ricordo che quella sera mio padre e mia madre erano tornati al lavoro [...] Io li guardavo dal balcone non capivo cosa era successo e gli ho detto che il papà di Pippi era tornato con una nave dai Caraibi. Loro mi hanno detto che le 
la finalità di Nove, in tal caso, è di far rivivere nella memoria collettiva la gravità di quel momento storico, anche se il narratore si mostra apparentemente poco sensibile al diverso valore dei due fatti, l'uno immaginario (il ritorno del padre di Pippi Calzelunghe) e l'altro reale (l'uccisione di Moro). La memoria collettiva e individuale acquistano nel romanzo una valenza ben più profonda rispetto a Woobinda, sono presenti e funzionali all'io narrante grazie al racconto omodiegetico. I personaggi sono calati nella realtà quotidiana, hanno un lavoro, agiscono in una determinata località, Milano o Santo Domingo, e la loro storia è pervasa da avvenimenti di scarsa importanza. La televisione ha un ruolo legato al passato e al presente, e inserito in un contesto futuro, la città di Milano, dove Michele porterà la sua sposa dominicana Francis. Seppure sotto forma diversa rispetto a Woobinda, Nove riprende la «cultura dello zapping», la brevità dei capitoli e la varietà di argomenti senza perdere di vista la story. Il ricordo delle trasmissioni degli anni '70 e '8o è completato dal ricordo della pubblicità: il Carrarmato, le caramelle Brancamenta, l'Allegro chirurgo, l'Ovomaltina. Anche il linguaggio, se associato a essa, cambia registro, diventa più anonimo e ne rispetta il canone espressivo ("dentro il Toblerone tradizionale ci trovi zucchero, burro di cacao, latte intero in polvere, pasta di cacao, lattosio, grasso butirrico, ecc.», Nove, 1997, p. 46) anche quando si riferisce a contesti a noi più contemporanei («l'amore, tra un uomo e una donna, è anche comprare uno specchio all'Ikea che sta su con i feltrini adesivi davanti e di dietro che costano 4500 a bustina», ibid., p. 17). La pubblicità invade la realtà di Puerto Plata, ed ecco la descrizione dei prodotti del supermarket caratterizzata dall'anadiplosi e da altre forme di ripetizione: "Da Silverio Messon trovi...», «Da Silverio Messon sono in vendita...", Michele, uomo senza qualità e dalla personalità catatonica, gestisce con difficoltà i rapporti interpersonali, e nelle sue scelte subisce il condizionamento del consumismo. Il supermercato è infatti il primo posto che visita dopo l'arrivo in città («forse per sentirmi un pochino a casa, in Svizzera o in Italia», ibid., p. 58) e trasferisce «alla meta turistica tutti i possibili veleni della contaminazione, dell'inquinamento, o quantomeno i parametri di una commercializzazione standard» (Barilli, 2000, p. II9). Il condizionamento pubblicitario televisivo, l'ansia di consumismo che pervade Michele si manifesta infine nella deludente considerazione che nel supermercato non si vendono le merendine del Mulino Bianco, l'alimento quotidiano indispensabile nella sua prima colazione a Milano.

Brigate Rosse lo avevano ucciso. Io in quel momento essendo piccolo avevo capito che le Brigate Rosse avevano ucciso il papà di Pippi Calzelunghe.» (Nove, 1997, p. 53.) 
Tra le fonti d'ispirazione si può individuare la soap opera televisiva Beautiful (Senardi, 2005, p. 56). Michele, parlando di Marina, la paragona infatti a Sheila e a Brooke. Beautiful entra direttamente in scena in uno dei primi appuntamenti di Michele con Francis, quando le parla dei protagonisti della fiction che acquista, di conseguenza, «una valenza criticoironica, diventa figura allo specchio di un romanzo abile a seminare false piste (il tema dell'amore, come rischio, caso, avventura, come un gratta e vinci) quasi a nascondere la sua natura segreta di indagine sui disastri della globalizzazione» (Senardi, 2005, p. 56). La seconda valenza della televisione è quella di essere, per Michele, occasione di fuga dalla realtà dominicana, ma anche strumento, occasione di divertimento collettivo e di aggregazione con la diretta della partita Juventus-Borussia, seguita da tutti i turisti italiani presenti a Puerto Plata.

Il romanzo successivo Amore mio infinito, anch'esso a narrazione omodiegetica, si divide geograficamente tra Viggiù e Milano. Il tempo narrativo va dai primi anni Settanta al 1999, e il contenuto è più autobiografico rispetto alle opere precedenti. Il protagonista, Matteo, un giovane di 28 anni che lavora in una ditta produttrice di banconi per il pesce, attraversa una crisi d'identità e nei 4 capitoli del romanzo ripercorre altrettante fasi della sua vita. Anche qui, come nel romanzo precedente, il protagonista opera una specie di autoanalisi attraverso la rivisitazione del passato, con un registro linguistico e un tono che cambiano a seconda dell'epoca trattata. La funzione mnestica assume, anche in questo caso, una valenza fondamentale, è costantemente presente nella story e fa entrare in questa ottica anche il ruolo della televisione. L'alienazione degli attanti di Woobinda e Superwoobinda è ormai lontana e sono rispettati i canoni tradizionali del romanzo. La disomogenea presenza della televisione è funzionale ai periodi narrati. Nel primo capitolo («La bambina. 1982») scritto in uno stile semplice e ironico, Matteo ricorda l'infanzia e la vita familiare trascorse a Viggiù. I riferimenti alla televisione, fatta eccezione per i programmi dell'infanzia o per il Festival di Sanremo che coinvolgono soprattutto il piccolo protagonista, tendono a sottolineare l'incomunicabilità degli adulti. La tv diventa un alibi per i genitori in crisi e ne allenta le tensioni, e chi ha poco o niente da dire commenta con il partner le notizie del telegiornale. In questo capitolo il linguaggio del romanzo si allontana da quello televisivo, tranne che in poche frasi derivate dalla pubblicità ("Quando due si amano mangiano sempre il Biancorì).

Nel secondo capitolo («I coccodrilli. I985») la funzione della televisione nella vita familiare si fa più dominante, e la programmazione televisiva condiziona ancor di più la vita dei componenti: Matteo, tredicenne, 
corteggia Maria raccontando le trame dei telefilm. Il mezzo televisivo si trasforma in uno scudo per difendersi dalla realtà e ostentare indifferenza quando, ad esempio, il padre annuncia al ragazzo la prossima morte della madre : «E ti aggrappi a rumore lontano della televisione, alla voce del presentatore dell'Almanacco del giorno dopo che diventa una casa dove abiti mentre fuori c'è la tempesta e tutto quello che non vuoi vedere» (Nove, 2000, p. 57); riempie il vuoto lasciato dalla sua morte: «Mia madre mi ha detto che finiva. Io la guardavo e sentivo che c'era e che era tutto ma dopo non ci sarebbe stata più e io e mio padre avremmo guardato la televisione in silenzio tutte le sere come due morti.» (Ibid., p. 65.)

Nel terzo capitolo («Primo bacio. 1987») il diciottenne Matteo, nervoso per il suo primo appuntamento con Silvia, si rilassa guardando i programmi di Frizzi e i telefilm di Happy Days. Parlare di Happy Days diventa anche l'occasione per sciogliere il ghiaccio con lei. Si accenna alla televisione nell'ultimo capitolo («Piazza Cordusio. I999») dove per Matteo ormai ventottenne, e neolaureato, il ricordo di alcuni programmi televisivi, come lo Zecchino d'oro o i primi programmi a colori, diventa l'occasione per rivivere alcuni momenti passati. In questo romanzo la televisione acquista, ancora una volta, una valenza multipla «quale canale comunicazionale, codice linguistico, luogo di formazione dell'immaginario, matrice del sistema simbolico e mitico [...] saldando in un nesso indicibile i due elementi del sistema nell'età della globalizzazione (comunicazione e merci)» (Mondello, 2007, p. I23).

$\mathrm{Ne}$ La più grande balena morta della Lombardia, romanzo suddiviso anch'esso in microepisodi, la narrazione oscilla tra memoria personale e fantasia. L'io narrante ripercorre, da adulto, come nei precedenti romanzi, l'infanzia e l'adolescenza vissuti negli anni Settanta a Viggiù. Il valore autobiografico dei microracconti (arricchito dall'esperienza «cannibale», dalla "cultura della merce») è esplicitamente dichiarato dallo scrittore in seconda di copertina: "Ho scritto La più grande balena morta della Lombardia per testimoniare innanzitutto a me stesso che ci sono altri mondi da raccontare. Sono tutti nella memoria. Noi li guardiamo da lontano.» (Nove, 2004, p. 2.) La «ricerca del tempo perduto» è scandita dal ritmo delle programmazioni televisive e dalla pubblicità. La televisione, spesso presente, sottolinea questa sensazione nostalgica con il ricordo di programmi che scandiscono il passaggio del tempo e accompagnano la memoria del protagonista: fungono da colonna sonora o da foto tratte dall'album di famiglia, fanno da sottofondo nella mente del narratore a situazioni famigliari rimarcate da eventi politici e di cronaca dell'epoca, oppure segnano il mutamento di costumi del Paese. Ne è un esempio 
l'episodio «L'invenzione della televisione a colori» in cui si descrive in modo felliniano l'eccitazione degli abitanti di Viggiù nel vedere il primo programma televisivo a colori, un evento già accennato nel romanzo precedente. La realtà dell'epoca è ricordata attraverso l'informazione televisiva, fatto che induce l'io narrante a riflettere su quanto il mondo degli adulti sia poco comprensibile a un bambino. Ne «Il giorno dell'arresto di Enzo Tortora» domanda a un immaginario extraterrestre, incontrato nella cantina di casa, che cosa voglia dire la frase proferita dalla madre ( $U \mathrm{Un}$ conto è la vita e un conto è Portobello»). Rivive i momenti dell'arresto di Toni Negri, i primi documentari sul Biafra e sull'olocausto, i delitti di mafia, la pubblicità della carne Montana e dell'Ovomaltina. La tv è un mostro nella fantasiosa descrizione dei malati del Cottolengo («c'era anche un uomo con al posto della testa un televisore e nel sangue gli scorrevano le trasmissioni», Nove, 2004, p. 88), ma può diventare anche un rifugio. Si deplorano gli alienanti programmi televisivi con cui gli adulti «si programmano la testa per vedere quello che vogliono loro esattamente come lo ha spiegato il telegiornale» (ibid., p. II4), fino a diventare come i robot nell'episodio «Tetsujin 28-Go e gli altri 22 robot scappati dal negozio di giocattoli». Rispetto ai due precedenti volumi di racconti la tv si configura in modo meno condizionante, sia per il differente approccio del rapporto letteratura-massmedia che Nove è andato maturando negli anni, sia per l'uso che del mezzo televisivo veniva fatto negli anni '70 e '80: non era solo una forma di intrattenimento del pubblico ma anche uno strumento d'informazione. Si trasforma anche il linguaggio, si fa più poetico, meno aggressivo e quasi per nulla dominato da espressioni e frasi dei format televisivi ${ }^{4}$.

Nove si allontana dai canoni del romanzo con il libro-inchiesta $M i$ chiamo Roberta, ho 42 anni, guadagno 250 euro al mese. Nel prologo alla «Storia di Riccardo», uno dei tanti episodi narrati, definisce (come è stato già riferito) il rapporto tra televisione e letteratura partendo proprio dagli anni Settanta, caratterizzati dalla «emersione rampante delle prime televisioni private» e dalla nascita della «cultura del trash» con «insostenibili, lunghissime trasmissioni a premi generose di formaggi in omaggio e di tettone sconosciute» (Nove, 2006, p. 4I) che costituivano la nuova alternativa all' «ingessata e triste televisione di Stato». Nove sostiene che la cultura del trash (che negli anni ha conquistato ampi spazi anche nella Rai)

4. Nel frattempo lo scrittore si era misurato in altre esperienze letterarie: la riedizione in chiave moderna del Candido di Voltaire, scritto con Andrea Liberovici; la pubblicazione delle sue raccolte poetiche composte dal I984 al 1996 e uscite nel 2003; la scrittura di poesie ispirate a canzoni rock in Nelle galassie oggi come oggi. 
giustifica l'ascesa e la caduta della letteratura $p u l p$, nata come testimonianza letteraria del nuovo modo di fare televisione. La sempre più bassa qualità dei programmi fa prevedere allo scrittore che prima o poi il pubblico intelligente prenderà le distanze dalla televisione, un concetto già anticipato nel racconto "Woobinda» dell'omonima raccolta: "Un giorno non ci saranno più foreste e allora Woobinda finirebbe come alla televisione di oggi, che non esiste proprio.» (Nove, I998, p. I5.)

Se il discorso sul precariato nelle reti televisive monopolizza la «Storia di Riccardo", in altri luoghi si ricordano le gag di Cochi e Renato sui terroni e i polentoni; si ironizza sul voler apparire a tutti i costi; si definisce la televisione una droga con tutte le sue virtù oppiacee. Gli intervistati concordano nel dichiarare che i programmi televisivi non offrono più informazione e che "la televisione è il sistema che ogni giorno ti cancella la storia, riduce il tempo all'oggi assoluto» (Nove, 2006, p. I25). Nell'ultimo capitolo Carlo si dichiara fortunato di non aver tempo di guardare la tv. Insomma, quello che in Woobinda, in Amore mio infinito e nella Più grande balena morta è la descrizione ironico-sarcastica della vita della provincia italiana negli anni Settanta condizionata negli orari e nelle abitudini dal mezzo televisivo, in Mi chiamo Roberta... diventa più che mai una dichiarazione esplicita della futilità delle trasmissioni televisive ormai utili solo a se stesse.

L'opera letteraria di Aldo Nove ha subito un notevole cambiamento stilemico nell'arco di un decennio. Nelle due prime raccolte di racconti era sua intenzione creare una realtà attanziale modellata dalla tv a dimostrazione di quanto possa influire la tecnologia dei mass media sulla nostra vita. La forma e i contenuti, ostentamente poco elaborati nei suoi racconti e romanzi, non mescolano soltanto tematiche letterarie e linguaggio ai paradigmi culturali e linguistici offerti dai palinsesti televisivi, dalla tv trash o dalla pubblicità, ma tendono a mettere in ridicolo quello che la società, ormai condizionata dai media, esibisce senza vergogna.

Molteplici sono i richiami teorico-letterari, già esaminati da altri (cito emblematicamente: Senardi, 2005; Schiavo, 200I). A essi Nove attinge per consolidare il suo punto di vista e sottolineare l'aspetto antropologico del condizionamento televisivo che tende a massificare gli individui. I suoi attanti sono, per lo più, espressione di una collettività ormai condizionata o dominata dal consumismo; la televisione è apocalitticamente posta alla stregua di una droga domestica, con un'inevitabile assuefazione dei protagonisti dei romanzi e racconti, spinti ad assumerne dosi sempre più massicce. La loro crescente alienazione, dagli anni Settanta ad oggi, si manifesta nella maggiore superficialità nei rapporti interpersonali e nella 
sempre più presente cultura della merce. Questo li induce ad adeguarsi ai miti della televisione e della pubblicità e, lascia intendere Nove, genera anche un comportamento violento nella vita quotidiana: lo scrittore sembra «rendere responsabile del dilagare della violenza proprio l'infausto mezzo, di cui la sensazione, il sesso e la violenza sono i tre ingredienti principali» (Serkowska, 2003, p. 475). Così, si risolvono a compiere il male con la massima spontaneità e senza alcuna esitazione. Aldo Nove, insomma, si sente portavoce delle speranze e delle attese deluse della sua generazione.

\section{Bibliografia}

Nove Aldo, Puerto Plata Market, Torino, Einaudi, 1997.

—, Superwoobinda, Torino, Einaudi, 1997.

—, Amore mio infinito, Torino, Einaudi, 2000.

-, Mi chiamo Roberta, ho 42 anni, guadagno 250 euro al mese..., Torino, Einaudi, 2006.

AA. VV., Gioventù cannibale, Torino, Einaudi, 1996.

Arcangeli Massimo, Giovani scrittori, scritture giovani, Roma, Carocci, 2007.

Barenghi Mario, Oltre il Novecento. Appunti su un decennio di narrativa (I988-1998), Milano, Marcos y Marcos, I999.

BARILli Renato, «Bianchi contro neri», $\grave{E}$ arrivata la terza ondata, Torino, Testo e Immagine, 2000.

CASAdei Alberto, Stile e tradizione del romanzo italiano contemporaneo, Bologna, Il Mulino, 2007.

Frasca Gabriele, La lettera che muore, Roma, Meltemi, 2005.

Gervasutti Luca, Dannati \& Sognatori. Guida alla nuova narrativa italiana, Pasian di Prato, Campanotto, 1998.

La Porta Filippo, La nuova narrativa italiana, Torino, Bollati Boringhieri, 2003.

Mondello Elisabetta (a cura di), La narrativa italiana degli anni Novanta, Roma, Meltemi, 2004.

—, In principio fu Tondelli, Milano, Il Saggiatore, 2007.

Ottonieri Tommaso, La plastica della lingua, Torino, Bollati Boringhieri, 2000.

Pignotti Lamberto, Scritture convergenti, Udine, Campanotto, 2005.

Schiavo Carlo, "Aldo Nove, Amore mio infinito», Bollettino 'goo, n. I, $200 I$. 
-, Aldo Nove, Fiesole, Cadmo, 2005.

Serkowska Hanna, "Il centone postmoderno», Kwartalnik neofilologiczny, vol. I, n. 4, 2003.

Sinibaldi Marino, Pulp, Roma, Donzelli, 1997. 